\title{
Una mirada a la investigación educativa en Chihuahua, a través de los estados de conocimiento
}

\author{
Rigoberto Martínez Escárcega \\ Sandra Vega Villarreal \\ Profesores investigadores \\ Centro de Investigación y Docencia \\ Universidad Pedagógica Nacional, Unidad 081
}

\section{Resumen}

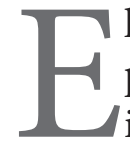

1 presente escrito contiene un informe preliminar sobre un diagnóstico de la investigación educativa realizado en el Estado de Chihuahua. La investigación en su conjunto contempla tres fuentes de información: los estados de conocimiento coordinados por la Secretaría de Educación, Cultura y Deporte; las actividades realizadas en el Congreso Nacional de Investigación Educativa organizado por el Consejo Mexicano de Investigación Educativa (COMIE); y la información obtenida a través de un instrumento que se diseñó a nivel nacional, tomando como base los análisis y las discusiones de la Red Mexicana de Investigadores de la Investigación Educativa (REDMIIE), el cual se aplicó a los agentes y a las instituciones de educación superior de la entidad. A pesar de la modestia heurística de esta trabajo, es el primer esfuerzo sistemático por dar cuenta del estado que guarda la investigación educativa en la entidad.

Palabras clave: diagnóstico, investigación, educación

\section{Introducción}

El presente documento contiene un informe preliminar sobre los resultados de un diagnóstico de la investigación educativa en el estado de Chihuahua. El estudio da cuenta de la producción generada en cinco dimensiones: área temática, tiempo, acercamiento metodológico, objeto y nivel educativo investigado. También se estudia a las instituciones, a los agentes y a las condiciones de publicación y difusión de la investigación educativa en la entidad. 
Esta investigación forma parte de un esfuerzo más amplio encabezado por el COMIE, para construir una visión integral sobre las condiciones institucionales en las que se realiza la investigación educativa a nivel nacional. El diagnóstico estatal es un objeto de estudio a partir del cual se han establecido relaciones sistemáticas de colaboración e intercambio académico entre la Red de Investigadores Educativos Chihuahua (REDIECH) y la REDMIIE.

La investigación educativa en México se ha desarrollado desde enfoques marcadamente centralizados. Los autores de las publicaciones de circulación nacional se encuentran, predominantemente, adscritos a instituciones ubicadas en el Distrito Federal. Prevalece la visión del desarrollo de la investigación educativa nacional a partir del centro. Es por ello, y en el contexto de la federalización de la educación, que cobra importancia conocer el desarrollo de la investigación educativa en los diferentes estados de la república mexicana.

Han pasado tres décadas desde que se inició la elaboración de los estados de conocimiento de la investigación educativa a nivel nacional, $y$ en algunas entidades federativas se han constituido equipos de trabajo que han publicado diagnósticos sobre la investigación educativa a nivel estatal. Con estos esfuerzos se han podido identificar las fortalezas y debilidades de la investigación educativa, así como evaluar las condiciones institucionales y el impacto de la investigación en la calidad de la educación.

En el estado de Chihuahua, en el 2006, se constituyeron varios equipos de trabajo, en torno a la convocatoria de la entonces Secretaría de Educación y Cultura, con la participación de un gran número de investigadores de diferentes instituciones de educación superior, que asumieron el reto de dar cuenta sobre los estados de conocimiento de la investigación educativa en la entidad.

Como resultado de la construcción de esos estados de conocimiento, se agenda como temática urgente, desarrollar un diagnóstico estatal que pueda dar cuenta de forma sistemática sobre las condiciones personales e institucionales en las que se produce investigación educativa. La REDIECH, en conjunto con el Gobierno del Estado de Chihuahua a través del Departamento de Investigación, ha asumido este reto.

Se plantean las siguientes preguntas de investigación que sirvieron como hilos conductores en la realización del proyecto: ¿Cuál es el estado que guarda la producción de investigación educativa en el estado de Chihuahua? ¿Cuáles son los agentes y los grupos de académicos que producen investigación educativa en Chihuahua? ¿Cuáles son las instituciones donde se produce investigación educativa en el estado de Chihuahua? ¿Cuáles son las condiciones para la publicación y difusión de la investigación educativa en el estado de Chihuahua?

El objetivo de la presente investigación es impulsar la realización de un diagnóstico de la investigación educativa a nivel estatal, que permita fortalecer la relación entre grupos y redes de académicos, así como propiciar el mejoramiento de las condiciones institucionales de los programas de posgrado que permitan formar investigadores de alto nivel. 


\section{Metodología empleada}

En este documento se presenta un análisis descriptivo de la investigación educativa en Chihuahua. Las fuentes de información para la elaboración del diagnóstico estatal fueron los estados del conocimiento estatales elaborados hasta el 2008, la participación de los investigadores en los Congresos Nacionales de Investigación Educativa organizados por el COMIE y, la información obtenida a través de un instrumento de investigación elaborado de forma específica por el equipo coordinador del proyecto.

Este proyecto de investigación comprende una etapa cuantitativa-descriptiva y otra etapa cualitativa-interpretativa. Aunque en este informe preliminar se presentan solo algunos datos estadísticos correspondientes a la primera etapa.

Para el desarrollo del diagnóstico se conformó un equipo de trabajo con investigadores adscritos a diferentes instituciones de educación superior de la entidad. Posteriormente se elaboró un instrumento para recabar la información tomando como base la discusión a nivel nacional que tuvo como escenario las reuniones de la REDMIIE.

El llenado del instrumento se llevó a cabo a través de internet, con acceso en la página oficial del Gobierno del Estado de Chihuahua. La difusión del proyecto se realizó a través de la publicación de una convocatoria que emitió el Departamento de Investigación de la Secretaría de Educación, Cultura y Deporte del Gobierno del Estado de Chihuahua, dirigida a todos los investigadores de la entidad -independientes o adscritos a alguna institución de educación superior-, que tuvieran producción dentro del ámbito educativo.

Finalmente se elaboró un informe de investigación, que permite tener parámetros de comparación con las demás entidades federativas del país.

\section{Discusión de resultados}

\section{Producción}

Se valoraron 458 productos en total, los cuales se distribuyeron en 10 áreas temáticas. La distribución de la producción por área temática es dispar, mientras que en el área de Historiografía de la educación se detectaron 135 productos, en el área de Crianza y de desarrollo humano se registraron ocho productos (tabla No. 1). La media de la producción por área temática es de 45.8 productos. Cuatro áreas temáticas se encuentran por encima de la media (Historiografía de la educación con 135 productos, Sujetos con 78 productos, Género con 56 productos y Currículum con 55 productos). Otras cuatro áreas se encuentran alejadas de la media con poca producción (Crianza y desarrollo humano con ocho productos, Aprendizaje y procesos psicológicos asociados con 17 productos, Prácticas educativas con 18 productos y Política educativa con 21 productos). Dos áreas se encuentran cerca de la media (Lenguas con 40 productos y La investigación educativa su epistemología y sus métodos con 30 productos). 
Tabla No. 1

\section{Producción por área}

\begin{tabular}{|c|c|}
\hline Área: & Producción: \\
\hline $\begin{array}{l}\text { Aprendizaje y procesos psicológicos } \\
\text { asociados }\end{array}$ & 17 \\
\hline Crianza y desarrollo humano & 8 \\
\hline Currículum & 55 \\
\hline Lenguas & 40 \\
\hline Historiografía de la educación & 135 \\
\hline $\begin{array}{l}\text { La investigación educativa, su } \\
\text { epistemología y sus métodos }\end{array}$ & 30 \\
\hline Género & 56 \\
\hline Política educativa & 21 \\
\hline Prácticas educativas & 18 \\
\hline Sujetos & 78 \\
\hline TOTAL & 458 \\
\hline
\end{tabular}

La distribución de la producción por área temática permite dar cuenta de forma descriptiva, cómo el desarrollo del campo de la investigación educativa en el estado de Chihuahua es desigual, mientras que en algunas áreas temáticas se tiene una producción constante y abundante, en otras áreas la producción es incipiente.

La producción se distribuyó temporalmente en periodos de cinco años. El primer periodo comprende la producción presentada antes de 1990. El último periodo comprende tres años, ya que la producción valorada llega hasta el 2008.

Al abordar la producción en la dimensión temporal, se puede apreciar de forma contundente una tendencia creciente. Mientras que en el periodo comprendido antes de 1990 se registraron 10 productos, en el periodo comprendido entre 2006 y 2008 se registraron 158 productos (Tabla No. 2).

A partir del periodo 1996-2000 la investigación educativa se desarrolla de forma significativa, ya que mientras en los periodos anteriores la producción creció un $100 \%$ en este periodo la producción registra un crecimiento mayor al 300\%. Estos datos permiten mostrar el impacto directo de los procesos de formación institucionalizados en la producción de investigación educativa, ya que en la década de los noventa se fundaron, en la entidad, las principales instituciones de posgrado en educación.

Tabla No. 2

Producción por tiempo

\begin{tabular}{|l|c|}
\hline \multicolumn{1}{|c|}{ Tiempo: } & Producción: \\
\hline Antes de 1990 & 10 \\
\hline De 1990 a 1995 & 22 \\
\hline De 1996 a 2000 & 78 \\
\hline De 2001 a 2005 & 165 \\
\hline De 2006 a 2008 & 158 \\
\hline Sin fecha & 25 \\
\hline TOTAL & $\mathbf{4 5 8}$ \\
\hline
\end{tabular}

Otra dimensión importante de la producción es la clasificación por el posicionamiento epistemológico y metodológico adoptado por los agentes de la investigación. Es importante señalar que el posicionamiento en cuantitativos, cualitativos y mixtos son asunciones realizadas por los autores de los trabajos analizados y no por la perspectiva teórica adoptada en la presente investigación. 
La mayor parte de los trabajos se realizaron a partir de un acercamiento metodológico cualitativo, mientras que una escasa minoría define su acercamiento metodológico como mixto. Es clara la hegemonía de los cualitativos sobre los cuantitativos (Tabla No. 3).

Tabla No. 3

Producción por método

\begin{tabular}{|l|c|}
\hline \multicolumn{1}{|c|}{ Método: } & Producción: \\
\hline Cualitativo & 338 \\
\hline Cuantitativo & 101 \\
\hline Mixto & 19 \\
\hline TOTAL & 458 \\
\hline
\end{tabular}

El predominio de los métodos cualitativos sobre los cuantitativos muestra cómo la investigación educativa en Chihuahua es de reciente aparición, alejada de la influencia positivista que predominó en el campo de las ciencias sociales durante el siglo XX.

Es importante analizar la producción por objeto investigado. Se puede observar que los procesos y los sujetos son el objeto de estudio más abordado por los agentes de la investigación educativa en la entidad. En cambio las ideas y las instituciones tienen un papel marginal en el campo (Tabla No. 4). Estos datos permiten dar cuenta de cómo los acercamientos empíricos en la investigación educativa predominan de forma significativa sobre las investigaciones de carácter teórico.
Tabla No. 4

Producción por objeto investigado

\begin{tabular}{|l|c|}
\hline \multicolumn{1}{|c|}{ Objeto investigado: } & Producción: \\
\hline Ideas & 39 \\
\hline Sujetos & 165 \\
\hline Instituciones & 59 \\
\hline Procesos & 195 \\
\hline TOTAL & 458 \\
\hline
\end{tabular}

Otra dimensión importante de la producción es el nivel educativo investigado. Se aprecia con facilidad que los niveles de educación básica y educación superior son los más investigados, mientras que los niveles de educación inicial y media superior, prácticamente están fuera del interés de los agentes de la investigación (Tabla No. 5).

Estos datos permiten inferir cuáles son los niveles educativos en donde las políticas públicas y los procesos de gestión escolar están más racionalizados.

Es importante resaltar cómo una gran parte de la producción que se registra en la entidad no tiene como objeto de estudio algún nivel educativo en particular. Esto se debe a que la mayor parte de la producción se encuentra en el área temática de Historiografía de la educación.

En la categoría de otro se encuentran las investigaciones que estudian los niveles de educación especial, educación para el trabajo y otros tipos y modalidades. Estos sectores del nivel educativo estatal se encuentran poco investigados. 
Tabla No. 5

Producción por nivel educativo investigado

\begin{tabular}{|l|c|}
\hline \multicolumn{1}{|c|}{$\begin{array}{c}\text { Nivel educativo } \\
\text { investigado: }\end{array}$} & Producción: \\
\hline Educación inicial & 8 \\
\hline Educación básica & 170 \\
\hline Educación media superior & 9 \\
\hline Educación superior & 98 \\
\hline Otro & 8 \\
\hline No aplica & 165 \\
\hline TOTAL & $\mathbf{4 5 8}$ \\
\hline
\end{tabular}

\section{Instituciones}

Se pueden identificar a diez instituciones en la entidad que tiene más de seis productos. La producción por institución está desequilibrada, por un lado hay instituciones que tienen 125 productos y por otro hay instituciones que registran menos de seis productos.

Cuatro instituciones se encuentran arriba de la media de producción, otras cuatro se encuentran significativamente debajo de la media y sólo dos se ubican cerca de la media (Tabla No. 6).

La producción por institución permite identificar la antigüedad de los programas de posgrado. Las instituciones con más producción son las primeras que implementaron un programa de posgrado (la UPN unidad Chihuahua, la UACH, el CID y el CCHEP). Por otro lado están algunas instituciones con posgrados de reciente creación y poca producción (UPN unidad Juárez, UPN unidad Parral, ITESM).

Hay dos instituciones que a pesar de que la investigación es parte de sus funciones sustantivas, por su naturaleza carecen de espacios para la formación de investigadores, y esto se ve reflejado en una baja producción (ByCENECH y SECD).

Tabla No. 6

\section{Producción por institución}

\begin{tabular}{|l|c|}
\hline \multicolumn{1}{|c|}{ Institución: } & Producción: \\
\hline $\begin{array}{l}\text { Universidad Pedagógica Nacional } \\
\text { Unidad Chihuahua }\end{array}$ & 125 \\
\hline $\begin{array}{l}\text { Universidad Pedagógica Nacional } \\
\text { Unidad Juárez }\end{array}$ & 10 \\
\hline $\begin{array}{l}\text { Universidad Pedagógica Nacional } \\
\text { Unidad Parral }\end{array}$ & 30 \\
\hline $\begin{array}{l}\text { Universidad Autónoma de } \\
\text { Chihuahua }\end{array}$ & 53 \\
\hline $\begin{array}{l}\text { Universidad Autónoma de Ciudad } \\
\text { Juárez }\end{array}$ & 43 \\
\hline $\begin{array}{l}\text { Centro de Investigación y } \\
\text { Docencia }\end{array}$ & 56 \\
\hline $\begin{array}{l}\text { Centro Chihuahuense de Estudios } \\
\text { de Posgrado }\end{array}$ & 47 \\
\hline $\begin{array}{l}\text { Benemérita y Centenaria Escuela } \\
\text { Normal del Estado de Chihuahua }\end{array}$ & 67 \\
\hline $\begin{array}{l}\text { Secretaría de Educación, Cultura } \\
\text { y Deporte }\end{array}$ & 48 \\
\hline $\begin{array}{l}\text { Instituto Tecnológico de Estudios } \\
\text { Superiores de Monterrey }\end{array}$ & \\
\hline Otros & \\
\hline TOTAL & \\
\hline
\end{tabular}

\section{Agentes de la investigación}

Otra dimensión importante del campo de la investigación educativa son los agentes. La clasificación de los agentes de la investigación educativa de acuerdo al número de pro- 
ductos valorados permite tener una visión más objetiva del campo.

Se valoraron 458 productos de 387 investigadores en total. Se registran 357 investigadores con un solo producto y sólo dos investigadores con más de seis productos (Tabla No. 7).

Se puede establecer una categorización de los investigadores educativos. En primer lugar se encuentran los agentes potenciales de la investigación, que tienen un producto de investigación. Con un solo producto no se pueden considerar investigadores en el pleno sentido de la palabra, pero posiblemente se conviertan en investigadores en un futuro inmediato. En segundo lugar se tiene a los agentes de reciente ingreso al campo de la investigación educativa con dos y tres productos. En tercer lugar se encuentran los investigadores en desarrollo con cuatro, cinco y seis productos. $\mathrm{Y}$ en cuarto lugar se ubican los investigadores consolidados con más de seis productos.

Tabla No. 7

Producción por investigador

\begin{tabular}{|l|c|}
\hline \multicolumn{1}{|c|}{ 2Producciones: } & Investigadores: \\
\hline Un producto & 357 \\
\hline Dos productos & 12 \\
\hline Tres productos & 7 \\
\hline Cuatro productos & 5 \\
\hline Cinco productos & 1 \\
\hline Seis productos & 3 \\
\hline Más de seis productos & 2 \\
\hline TOTAL & 387 \\
\hline
\end{tabular}

Se registran 357 agentes potenciales de la investigación educativa, 19 investigadores de reciente ingreso al campo, nueve investigadores en desarrollo y dos investigadores consolidados.

Esta dimensión permite dar cuenta del desarrollo incipiente del campo de la investigación educativa en el estado de Chihuahua. Se registra a no más de 30 agentes activos en el campo. El número tan escaso de agentes en el campo de la investigación educativa es un indicador del bajo nivel de racionalización del sistema educativo estatal.

Un problema fundamental en el campo de la investigación educativa en la entidad es la falta de oficio científico, la mayor parte de los agentes no están produciendo conocimiento científico de forma permanente.

Aunque, una fortaleza importante del campo de la investigación educativa es la generación de un gran potencial para la investigación, que en un plazo no muy lejano se puede convertir en una gran comunidad académica que influya de forma significativa sobre la racionalización del sistema educativo estatal.

\section{Publicación y difusión}

Por último se tiene un acercamiento al campo de la investigación educativa a partir del tipo de producto generado. Se valoraron libros, capítulos de libros, tesis de maestría, artículos, ponencias, cuadernos de trabajo y documentos electrónicos.

Los datos son contundentes, se registran 204 tesis de maestría de 458 productos valorados en total. Estos productos no fueron publicados ni debatidos en espacios académicos públicos. Sólo se realizaron con fines de acre- 
ditación. Esta producción está desvinculada del campo de la investigación educativa. No toma como punto de partida lo producido en el campo ni las necesidades del sistema educativo estatal.

Por otro lado se encuentra la producción publicada y difundida a nivel local o nacional. Entre libros, capítulos de libro, artículos y ponencias se registran 192 productos (Tabla No. 8).

Tabla No. 8

Producción por tipo de producto

\begin{tabular}{|l|c|}
\hline \multicolumn{1}{|c|}{ Tipo de producto: } & Producción: \\
\hline Libro & 61 \\
\hline Capítulo de libro & 12 \\
\hline Tesis de maestría & 204 \\
\hline Artículo & 89 \\
\hline Ponencia & 30 \\
\hline Otros & 62 \\
\hline TOTAL & $\mathbf{4 5 8}$ \\
\hline
\end{tabular}

La mayor parte de la producción publicada y difundida está en manos de los 30 agentes de la investigación con más de un producto en el campo. Mientras que las tesis de maestría pertenecen a los agentes con un solo producto registrado en el campo.

Las tesis de maestría, por su volumen, distorsionan o alteran los datos acerca de la investigación del campo de la investigación educativa en la entidad. La mayor parte de los agentes con una sola producción son egresados de maestría que solo tienen un producto, motivados por un interés inmediato de adquirir el grado, sin pretensiones de ejercer el oficio de investigador.
No obstante, los 192 productos publicados son un dato alentador que da cuenta del surgimiento del campo de la investigación educativa en Chihuahua, el cual empieza a llamar la atención de la comunidad académica a nivel nacional e internacional.

\section{Conclusiones}

Lo primero que habría que hacer notar es que la producción en el campo de la investigación educativa no es homogénea; mientras que el área temática de Historiografía contempla 135 productos el área temática de Crianza y desarrollo humano sólo tiene seis investigaciones. Es importante impulsar el desarrollo de la investigación educativa en todas las áreas temáticas.

El enfoque metodológico que predomina en la investigación educativa es el cualitativo, este dato es un indicador de lo reciente que es esta actividad, alejada del enfoque cuantitativo que predominó en el siglo XX. Aunque no se deben descuidar los distintos acercamientos metodológicos a los problemas educativos de la entidad.

Los objetos más estudiados en la entidad son los Sujetos y los Procesos educativos. Ocupando un papel marginal las ideas y las instituciones. Este es un indicador de que predomina la investigación empírica sobre la investigación en el campo de la teoría. Se debe enfatizar la importancia de la investigación básica, que permite un desarrollo del conocimiento.

Los niveles educativos más investigados son educación básica y educación superior, dejando prácticamente descuidados todos los demás niveles y modalidades del sistema estatal de educación. 
Los productos de la investigación educativa se aglutinan en las instituciones de posgrado con mayor antigüedad. Por lo que es importante apoyar a las instituciones existentes y seguir impulsando la creación de nuevos espacios en donde se formen investigadores educativos.

En el periodo objeto de estudio se analizaron una cantidad importante de productos de investigación, sin embargo la mayoría corresponde a tesis de maestría. Por lo que se puede identificar a no más de 30 agentes activos en el campo de la investigación educativa en Chihuahua. Se debe incentivar de forma creativa la producción de investigación educativa para que los agentes potenciales se conviertan en investigadores desarrollados que ayuden a racionalizar el sistema educativo estatal.

\section{Fuentes de información}

García Sánchez, Rafael (2010) Prácticas educativas en espacios escolares. Estado de conocimiento de la investigación educativa en el estado de Chihuahua, tomo 9, México, Gobierno del Estado de Chihuahua.

Hernández Orozco, Guillermo (2010) Historiografía de la educa- ción. Estado de conocimiento de la investigación educativa en el estado de Chihuahua, tomo 5, México, Gobierno del Estado de Chihuahua.

Hinojosa Luján, Romelia (2010) Miradas a la educación desde el género. Estado de conocimiento de la investigación educativa en el estado de Chihuahua, tomo 7, México, Gobierno del Estado de Chihuahua.

Jurado Campusano, Norma (2010) Política educativa. Estado de conocimiento de la investigación educativa en el estado de Chihuahua, tomo 8, México, Gobierno del Estado de Chihuahua.

Lara García, Yolanda Isaura (2010) Crianza y desarrollo humano. Estado de conocimiento de la investigación educativa en el estado de Chihuahua, tomo 2, México, Gobierno del Estado de Chihuahua.

Madrigal Luna, Josefina (2010) Aprendizaje y procesos psicológicos asociados. Estado de conocimiento de la investigación educativa en el estado de Chihuahua, tomo 1, México, Gobierno del Estado de Chihuahua.

Maldonado Payán, Juan Carlos (2010) El currículum. Estado de conocimiento de la investigación educativa en el estado de Chihuahua, tomo 2, México, Gobierno del Estado de Chihuahua.

Martínez Escárcega, Rigoberto (2010) La investigación educativa, su epistemología y sus métodos. Estado de conocimiento de la investigación educativa en el estado de Chihuahua, tomo 6, México, Gobierno del Estado de Chihuahua.

Sandoval Arana, Jorge (2010) Sujetos de la educación. Estado de conocimiento de la investigación educativa en el estado de Chihuahua, tomo 10, México, Gobierno del Estado de Chihuahua..

Vega Villarreal, Sandra (2010) El campo de lenguas en la investigación educativa. Estado de conocimiento de la investigación educativa en el estado de Chihuahua, tomo 4, México, Gobierno del Estado de Chihuahua. 